\title{
ANALYTIC AXISYMMETRIC DYNAMICAL MODELS WITH THREE INTEGRALS OF MOTION
}

\author{
Herwig Dejonghe \& Tim de Zeeuw \\ The Institute for Advanced Study, Princeton, NJ 08540
}

We consider a family of inhomogeneous axisvmmetric mass models with a simple gravitational potential originally introduced by Kuzmin (1956):

$$
V_{S}=-\frac{G M}{\left[R^{2}+z^{2}+a^{2}+c^{2}+2 \sqrt{a^{2} c^{2}+c^{2} R^{2}+a^{2} z^{2}}\right]^{1 / 2}},
$$

where $R^{2}=x^{2}+y^{2}, a$ and $c$ are parameters, $G$ is the gravitational constant, and $M$ is the total mass of the corresponding density distribution.

The potential (1) and the density $\rho$ are most elegantly expressed in terms of prolate spheroidal coordinates $(\lambda, \phi, \nu)$ (cf. de Zeeuw 1985):

$$
V_{S}=-\frac{G M}{\sqrt{\lambda}+\sqrt{\nu}}
$$

and

$$
\rho=\frac{M c^{2}}{4 \pi} \frac{\lambda \nu+a^{2}(\lambda+3 \sqrt{\lambda \nu}+\nu)}{(\lambda \nu)^{3 / 2}(\sqrt{\lambda}+\sqrt{\nu})^{3}} .
$$

The surfaces of constant density are smooth and not too far from ellipsoidal for moderate values of the central axis ratio. The axis ratio increases slightly with increasing radius. The spherical limit is Hénon's (1960) isochrone.

As already shown by Kuzmin and Kutuzov (1962), the density distribution $\rho(R, z)$ can be written explicitly as $\rho\left(R, V_{S}\right)$. This fact makes it possible to use a standard inversion technique (Lynden-Bell 1962; Hunter 1975; Kalnajs 1976; Dejonghe 1986) in order to obtain in closed form - as a series of generalized hypergeometric functions-the unique distribution function $f\left(E, L_{z}{ }^{2}\right)$ that depends only on the two classical integrals of motion (the energy $E$ and the component $L_{z}$ of the angular momentum parallel to the symmetry axis) and that is consistent with the density. This $f$ is nonnegative for all oblate models in the sequence. Numerical tests indicate that $f \geq 0$ for prolate models only in case the central axis ratio is larger than about 0.7 . The velocity dispersions can be written in terms of elementary functions.

The potential of all these models is of Stäckel form, so that all orbits in them enjoy an exact third integral of motion $I_{3}$, which can be regarded as a generalization 
of the total angular momentum integral of the spherical limit (de Zeeuw \& LyndenBell 1985). We have developed a new method for the analytical construction of distribution functions $f\left(E, L_{z}{ }^{2}, I_{3}\right)$, and apply it to these mass models.

We write $f$ as the sum of two parts: $f\left(E, L_{z}^{2}, I_{3}\right)=f_{1}\left(E, L_{z}^{2}, I_{3}\right)+f_{2}\left(E, L_{z}^{2}\right)$, where $f_{1}$ is of the form

$$
f_{1}\left(E, L_{z}^{2}, I_{3}\right)=\sum_{\ell, m, n} c_{\ell m n} E^{\ell} L_{z}^{2 m}\left(p+q E+r L_{z}^{2}+s I_{3}\right)^{n}
$$

where $c_{\ell m n}, p, q, r$ and $s$ are parameters, and $\ell, m$ and $n$ are integers. This $f_{1}$ can be integrated fairly easily over velocity space, and results in a density $\rho_{1}$. The remaining density $\rho_{2}=\rho-\rho_{1}$ is then reproduced by $f_{2}$, which is obtained by the standard inversion technique. This produces - for the first time-exact analytic distribution functions for realistic axisymmetric models that depend on all three integrals of motion. The velocity dispersions in these models can again be given explicitly.

Not all values of the parameters in $f_{1}$ produce positive distribution functions. However, fine-tuning is possible, and leads to an interesting variety of anisotropic models with positive distribution functions. In particular, we have constructed models with $m=0, p=0, q=0$ and $r=s / 2$. In the spherical limit our distribution functions reduce to the form $f=f_{1}\left(E, L^{2}\right)+f_{2}(E)$, where $L$ is the total angular momentum.

\section{REFERENCES}

Dejonghe, H., 1986. Physics Reports, 133, 217.

de Zeeuw, P.T., 1985. Mon. Not. R. astr. Soc., 216, 273.

de Zeeuw, P.T. \& Lynden-Bell, D., 1985. Mon. Not. R. astr. Soc., 215, 713.

Hénon, M., 1960. Ann. d'Astrophys., 23, 474.

Hunter, C., 1975. Astron. J., 80, 783.

Kalnajs, A., 1976. Astrophys. J., 205, 751.

Kuzmin, G.G., 1956. Astr. Zh., 33, 27.

Kuzmin, G.G., \& Kutuzov, S.A., 1962. Bull. Abastumani Astroph. Obs., 27, 82.

Lynden-Bell, D., 1962. Mon. Not. R. astr. Soc., 123, 447. 